\title{
Analyse des retombées intermédiaires d'un dispositif de développement professionnel sur le soutien du développement langagier en centre de la petite enfance
}

Lisandre Bergeron-Morin

Université Laval

Christine Hamel

Université Laval

Caroline Bouchard

Université Laval

\section{Résumé}

La présente étude décrit la mise à l'essai d'un dispositif de développement professionnel quant au soutien du développement langagier offert aux enfants en centre de la petite enfance. Le dispositif, qui inclut une modalité de cointervention avec une orthophoniste, a été proposé à quatre éducatrices. À l'aide d'un devis mixte et à partir du modèle logique de 
Coldwell et Simkins (2011), les retombées intermédiaires du dispositif ont été analysées, à savoir les réactions des éducatrices, les nouvelles connaissances qu'elles ont acquises et les habiletés développées, ainsi que les changements dans leurs pratiques de soutien du développement langagier. Une triangulation entre les données sur la fréquence d'utilisation de quinze pratiques de soutien du développement langagier et l'analyse d'entretiens avec les éducatrices souligne l'importance d'ancrer ces nouvelles connaissances et habiletés dans le quotidien des éducatrices, et permet de mieux comprendre certains défis inhérents aux transformations de pratiques.

Mots-clés : développement professionnel, éducation à la petite enfance, pratiques de soutien du développement langagier, modèle logique, collaboration interprofessionnelle

\begin{abstract}
This study documents the implementation of a professional development initiative to support language development for children in early childhood centres. The initiative, which includes a co-intervention modality with a speech-language pathologist, was offered to four educators. Using a mixed design and the Coldwell and Simkins (2011) logic model, the intermediate outcomes of the system are analyzed, namely the educators' reactions, the new knowledge they acquired and the skills they developed, as well as the changes in their practices to support language development. A triangulation between data on the frequency of use of fifteen language development support practices and the analysis of interviews with educators underlines the importance of anchoring this new knowledge and skills in educators' daily lives and provides a better understanding of certain challenges inherent to changes in practices.
\end{abstract}

Keywords: early childhood education and care, professional development, language development supporting practices, logic model, interprofessional collaboration 


\section{Remerciements}

Cette étude a été rendue possible grâce à une bourse de recherche doctorale du Conseil de recherche en sciences humaines du Canada (CRSH). Un merci particulier aux quatre éducatrices, à leurs CPE et aux enfants de leurs groupes pour la réalisation de cette étude. 


\section{Introduction}

De nombreux écrits scientifiques portent sur le développement professionnel des éducatrices en petite enfance, particulièrement en ce qui concerne le soutien du développement du langage chez le jeune enfant (Schatcher, 2015). De fait, les habiletés langagières consolidées au cours de la période de la petite enfance, à travers les interactions quotidiennes, constituent un facteur déterminant de la réussite éducative des enfants (Dickinson, 2011 ; Duncan et al., 2007 ; Justice et al., 2018). Durant cette période, les éducatrices ont une position privilégiée auprès des enfants, puisqu'elles ont de multiples occasions d'utiliser au quotidien des pratiques de soutien du développement langagier (Dickinson, 2011). Abondamment décrites dans différentes recherches (p. ex., Barnes et Dickinson, 2017 ; Dockrell et al., 2015 ; Girolametto et Weitzman, 2002), ces pratiques font d'abord référence à des attitudes centrées sur les enfants telles que se mettre à leur hauteur, ajuster le rythme de la parole et des échanges à celui des enfants, et suivre leurs initiatives. Les éducatrices peuvent par la suite prolonger les échanges verbaux dans le groupe à l'aide de commentaires ou de questions, par exemple. Enfin, elles peuvent enrichir les conversations grâce, entre autres, à des reformulations des énoncés des enfants, par l'ajout d'explications ou par l'emploi d'un langage décontextualisé.

Pourtant, en dépit de connaissances grandissantes sur les pratiques optimales pour soutenir le développement langagier des enfants en service de garde éducatif, des écarts dans leur utilisation persistent entre les éducatrices et selon les milieux de garde (Bouchard et al., 2010 ; Justice et al., 2018 ; Piasta et al., 2012). Ainsi, les enfants n'ont pas tous accès à la même qualité de soutien du développement langagier (Burchinal, 2018). Ces inégalités affectent particulièrement les enfants qui proviennent de milieux socioéconomiques défavorisés (LoCasale-Crouch, 2007), où l'on retrouve, en raison d'une exposition accrue à différents facteurs de risque (p. ex., faible scolarité des parents, antécédents familiaux de difficultés d'apprentissage, contexte de monoparentalité), davantage d'enfants vulnérables sur le plan langagier (Hoff, 2003 ; Sylvestre et Mérette, 2012).

C'est pourquoi il existe un consensus parmi les experts quant à l'importance de favoriser, via la mise en place de dispositifs de développement professionnel auprès 
d'éducatrices, l'utilisation de pratiques reconnues comme efficaces pour soutenir le développement langagier des enfants (Schatcher et al., 2019 ; Zaslow et al., 2010).

\section{Des critères d'efficacité pour les dispositifs de développement professionnel}

De nombreux dispositifs ${ }^{1}$ de développement professionnel ont été mis en œuvre en matière de soutien du développement langagier (voir à ce sujet la recension des écrits de Schatcher, 2015). Deux exemples souvent cités parmi ces dispositifs sont Exceptional Coaching for Early Language and Literacy (ExCELL ; Wasik et Hindman, 2011) et Apprendre à parler avec plaisir (Learning Language and Loving It [LLLI], Girolametto et al., 2006). Dans le dispositif ExCELL, quatre demi-journées de formation sont offertes à des enseignantes en petite enfance sur une période de neuf mois. Ces demi-journées sont ponctuées de rencontres d'accompagnement hebdomadaires par un expert (Wasik et Hindman, 2013). Pour sa part, LLLI consiste, dans sa version intégrale, en quinze rencontres de formation avec des éducatrices, réparties sur un an, combinées à des séances d'accompagnement individualisé utilisant la vidéorétroaction (Girolametto et al., 2006).

De tels dispositifs de développement professionnel répondent aux critères d'efficacité du développement professionnel qui ressortent dans différents écrits (Dunst et Trivette, 2009 ; Fukkink et Lont, 2007 ; Peleman et al., 2018, Schachter et al., 2019 ; Wayne et al., 2008 ; Zaslow et al., 2010). Tant LLLI qu'ExCELL intègrent des activités d'apprentissage actif qui sollicitent la participation des éducatrices et permettent de leur offrir des rétroactions individuelles (Wayne et al., 2008). Ainsi, elles tissent des liens plus aisément entre les connaissances présentées et leur mise en pratique auprès des enfants au quotidien. Dans les deux dispositifs, le contenu s'appuie sur des assises théoriques, ce qui permet de favoriser la compréhension des processus à l'origine des pratiques de qualité (Schachter et al., 2019 ; Zaslow et al., 2010). Les activités s'échelonnent sur une période suffisante, près d'un an dans les deux cas, et sont assez intensives pour soutenir des transformations durables dans les pratiques. Notons cependant qu'il n'existe pas encore

1 Le terme dispositif réfère ici à un agencement de différentes modalités de développement professionnel (p. ex., formation, accompagnement, etc.) qui, par leur synergie, visent à entrainer des retombées sur les pratiques professionnelles 
de consensus quant à la durée et à l'intensité requises (Markussen-Brown et al., 2017). Notamment, parmi les 25 études incluses dans leur méta-analyse, Markussen-Brown et al. (2017) rapportent que le nombre d'heures de développement professionnel, soit la durée, peut varier de $6 \mathrm{~h}$ à $450 \mathrm{~h}$. Fait à noter, chaque heure supplémentaire d'accompagnement augmente les retombées potentielles sur les pratiques des éducatrices.

Cependant, ils précisent que l'arrimage de plusieurs modalités de développement professionnel s'avère encore plus important que la durée et l'intensité, puisque cela permettrait de mieux rejoindre les différents styles d'apprentissages des participants (Markussen-Brown et al., 2007). En ce sens, ExCELL s'axe sur deux modalités de développement professionnel, soit la formation et l'accompagnement dans le groupe. LLLI met plutôt l'accent sur les rencontres de formation, en y intégrant des séances de vidéorétroactions. Ces modalités permettent notamment de mobiliser la pratique réflexive chez les participantes (Dunst et Trivette, 2009 ; Schachter et al., 2019).

En respectant ces critères d'efficacité, des études ont effectivement démontré les retombées positives d'ExCELL et de LLLI sur le soutien du développement langagier offert par les éducatrices (Girolametto et al., 2006 ; Wasik et Hindman, 2014). Une étude sur LLLI conclut notamment à une augmentation significative de l'utilisation des pratiques qui permettent de prolonger les interactions verbales chez les participantes (Piasta et al., 2012). Girolametto et al. (2006) ont également observé des effets sur la participation verbale des enfants lors de conversations, sur la diversité de leur vocabulaire et sur le nombre d'interactions avec les pairs (Girolametto et al., 2006). Dans le même ordre d'idées, les enfants des groupes ayant participé à ExCELL amorcent et prolongent davantage les échanges et y utilisent un vocabulaire plus diversifié (Wasik et Hindman, 2011, 2014). Ces résultats confirment l'intérêt du développement professionnel des éducatrices en la matière.

\section{Des constats nuancés}

Cependant, ces constats positifs quant aux retombées des dispositifs de développement professionnel doivent être nuancés (Markussen-Brown et al., 2007, Piasta et al., 2012 ; Wasik et Hindman, 2014). De l'avis même des chercheurs qui ont déployé ExCELL et LLLI, ces dispositifs sont complexes à mettre en œuvre et difficiles à déployer à grande échelle, en raison des ressources humaines et financières nécessaires (Piasta 
et al., 2012 ; Wasik et Hindman, 2014). Lorsque des adaptations sont proposées, en limitant par exemple le temps accordé à de l'accompagnement individualisé, il s'avère ardu d'observer des changements dans les pratiques des éducatrices (McDonald et al., 2015 ; Scarinci et al., 2015). De plus, les retombées demeurent modestes en regard des efforts investis (Piasta et al., 2012 ; Wasik et Hindman, 2014). Wasik et Hindman (2014) expliquent à cet égard que les pratiques de soutien du développement langagier s'avèrent souvent complexes à transformer, car elles obligent l'éducatrice à revoir son rôle dans ses interactions auprès des enfants.

Sur le terrain, le développement professionnel des éducatrices se concrétise encore généralement par une offre de formations ponctuelles (Schachter et al., 2019), une modalité pourtant reconnue comme étant peu efficace (Peleman et al., 2018 ; Zaslow et al., 2010). Ce modus opérandi s'explique par des considérations financières et organisationnelles : les formations ponctuelles sont peu couteuses et les éducatrices peuvent facilement y assister en dehors des heures de travail (Schachter et al., 2019).

En regard de ces constats, il s'avère nécessaire de poursuivre les recherches afin de développer des dispositifs plus écologiques, c'est-à-dire qui s'intègrent mieux au contexte des éducatrices, et aux réalités financières et humaines des milieux, tout en s'inspirant du déploiement de dispositifs de développement professionnel bien documentés comme ExCELL et LLLI. C'est ce qui constitue l'objet de la présente étude.

\section{Un modèle logique pour l'évaluation d'un dispositif de développement professionnel}

Coldwell et Simkins (2011) ont proposé un modèle logique pour structurer l'évaluation du développement professionnel qui vise à décrire, par une logique d'effets en cascade, comment un dispositif de développement professionnel peut entrainer des changements dans le développement des enfants. Comme le montre la Figure 1, ces auteurs proposent de commencer par l'analyse de retombées dites intermédiaires, à savoir : (1) la réaction des participants, qui fait référence à l'appréciation des éducatrices ; (2) les apprentissages réalisés et leur développement personnel, c'est-à-dire les nouvelles connaissances que les participants ont développées, tant sur le plan théorique que pratique ; (3) les changements dans leurs comportements, à savoir si les changements observés dans leurs connaissances se traduisent effectivement par des changements dans leurs pratiques éducatives. 
Une meilleure compréhension de ces premiers paliers permet par la suite d'aborder l'évaluation des retombées finales, notamment sur le développement des enfants. Ce modèle logique tient compte du caractère hautement contextualisé des dispositifs de développement professionnel, c'est-à-dire de l'influence d'antécédents des participants et de facteurs modérateurs, comme le contexte de mise en œuvre et le soutien de l'organisation (Coldwell et Simkins, 2011 ; Coldwell, 2019 ; Leithwood et Levin, 2005).

\section{Figure 1}

\section{Modèle logique de Coldwell et Simkins}

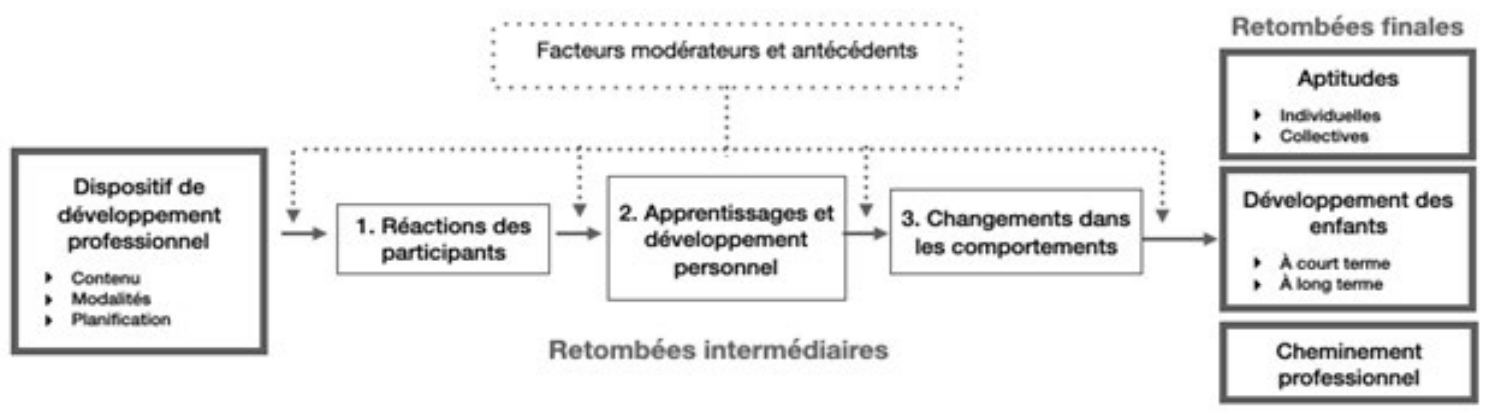

Note. Traduction libre de Coldwell et Simkins (2011).

\section{La présente étude}

La présente étude vise à décrire, par l'entremise du modèle logique de Coldwell et Simkins (2011), la mise à l'essai d'un dispositif de développement professionnel portant sur le soutien du développement langagier des enfants. Le dispositif proposé intègre une modalité de cointervention entre des éducatrices et une orthophoniste, une pratique émergente au Québec (Bergeron-Morin, 2017 ; Sylvestre et al., 2016), qui a le potentiel de s'arrimer à la réalité des mandats respectifs des orthophonistes et des éducatrices.

Les questions de recherche suivantes sont posées :

1. Quelles sont les retombées de la participation des éducatrices au dispositif de développement professionnel sur :

a. leurs réactions ?

b. leurs connaissances et habiletés quant au soutien du développement langagier? 
c. les pratiques de soutien du développement langagier mises en place dans leur quotidien?

2. Comment les éducatrices expliquent-elles l'influence des antécédents et des facteurs modérateurs sur les retombées intermédiaires ?

\section{Méthode}

\section{Les participantes}

Quatre éducatrices ont participé au dispositif de développement professionnel, après avoir manifesté leur intérêt à la suite d'une invitation de leur direction. Elles proviennent de deux centres de la petite enfance (CPE) desservant des populations défavorisées sur le plan social et matériel (Pampalon et al., 2004) et travaillent auprès de groupes multiâges de huit enfants. À partir de questionnaires remplis par chaque éducatrice, le Tableau 1 présente les caractéristiques des participantes, de même que la composition de leur groupe d'enfants.

\section{Tableau 1}

Caractéristiques des participantes et des enfants de leur groupe

\begin{tabular}{|c|c|c|c|c|c|c|}
\hline & \multirow{2}{*}{$\begin{array}{l}\text { Expérience } \\
\text { de travail }\end{array}$} & \multirow[b]{2}{*}{ Formation initiale } & \multirow{2}{*}{$\begin{array}{c}\text { Formation continue et } \\
\text { complémentaire }\end{array}$} & \multicolumn{3}{|c|}{ Enfants dans le groupe } \\
\hline & & & & $\mathrm{Nb}^{\mathrm{re}}$ & $\begin{array}{l}\text { Âge } \\
\text { (mois) }\end{array}$ & $\begin{array}{l}\text { Étendue } \\
\text { (mois) }\end{array}$ \\
\hline $\mathrm{P} 1$ & 25 ans & $\begin{array}{c}\text { DEC en éducation à } \\
\text { l'enfance }\end{array}$ & Certificat en pédagogie & 8 & 36.3 & $22-51$ \\
\hline P2 & 1 an & $\begin{array}{c}\text { AEC en éducation à } \\
\text { l'enfance }\end{array}$ & - & 8 & 40.7 & $31-55$ \\
\hline P3 & 15 ans & $\begin{array}{c}\text { DEC en éducation à } \\
\text { l'enfance }\end{array}$ & $\begin{array}{c}\text { AEC en stimulation du } \\
\text { langage }\end{array}$ & 8 & 39.4 & $22-61$ \\
\hline P4 & 30 ans & $\begin{array}{l}\text { DEC en éducation à } \\
\text { l'enfance }\end{array}$ & - & 8 & 39.9 & $32-53$ \\
\hline
\end{tabular}

Note. DEC : Diplôme d'études collégiales (3 ans) ; AEC : Attestation d'études collégiales (1 an) ; Certificat : formation universitaire (1 an). 


\section{Le dispositif de développement professionnel proposé}

Les caractéristiques du dispositif de développement professionnel proposé aux éducatrices sont décrites selon son contenu, ses modalités et sa planification (Coldwell et Simkins, 2011).

Contenu. Le contenu repose sur la présentation de pratiques de soutien du développement langagier regroupées selon trois catégories proposées par Girolametto et Weitzman (2002) : (a) pratiques pour se centrer sur les enfants (p. ex., suivre leur intérêt, ajuster le rythme de l'échange à celui des enfants, etc.) ; (b) pratiques pour promouvoir les interactions verbales (p. ex., commenter, questionner); (c) pratiques pour enrichir le langage (p. ex., reformuler, expliquer, utiliser un langage décontextualisé). L'accent a été mis sur la compréhension de ces pratiques, leur mise en œuvre et sur les façons de les ajuster en fonction de chaque enfant. Ce contenu est précis et spécifique (Fukkink et Lont, 2007).

Modalités. Le dispositif de développement professionnel inclut différentes modalités, circonscrites dans quatre volets (Dunst et Trivette, 2009), qui visent un engagement actif et une pratique réflexive chez les participantes (Wayne et al., 2008) :

1. Introduction : l'orthophoniste a offert une formation de trois heures sur les pratiques de soutien du développement langagier à l'ensemble des éducatrices du CPE. À la suite de la formation, chaque éducatrice a déterminé les objectifs qu'elle souhaitait poursuivre parmi les pratiques de soutien du développement langagier répertoriées (Zaslow et al., 2010).

2. Mise en pratique : six rencontres d'une heure de cointervention orthophoniste-éducatrice ont été proposées dans leur groupe. La cointervention vise à offrir un espace de collaboration au sein des routines quotidiennes en CPE, où tant l'expertise de l'orthophoniste que celle de l'éducatrice sont mises à profit pour observer les enfants du groupe et mettre à l'essai des pratiques auprès d'eux.

3. Réflexion : six sessions de rétroactions de 30 minutes avec l'orthophoniste ont suivi chaque rencontre dans le groupe. Ces sessions ont pour objectif de porter un regard réflexif sur les pratiques des éducatrices et de l'orthophoniste (Schachter et al., 2019). 
4. Réinvestissement : des périodes de réinvestissement dans le quotidien par les éducatrices se sont échelonnées entre les rencontres.

Planification. La formation et les six rencontres de cointervention dans le groupe se sont déroulées sur une période de trois mois, ce qui est réaliste dans le contexte professionnel des éducatrices et des orthophonistes. Il s'agit donc d'un dispositif de courte durée, mais à caractère intensif (Schachter, 2015).

\section{La collecte et l'analyse des données}

Un devis mixte simultané avec triangulation a été retenu (Corbière et Larivière, 2014), c'est-à-dire que les données qualitatives et quantitatives ont été recueillies de façon concomitante et intégrées au niveau de l'analyse. Le Tableau 2 présente les outils de collecte de données associés à chaque composante du modèle logique de Coldwell et Simkins (2011) analysée dans l'étude.

\section{Tableau 2}

Outils de collecte de données

\begin{tabular}{|c|c|}
\hline $\begin{array}{l}\text { Composantes du modèle de } \\
\text { Coldwell et Simkins (2011) }\end{array}$ & Outils de collecte de données \\
\hline Dispositif de développement professionnel & $\begin{array}{l}\text { Journal de bord de l'orthophoniste } \\
\text { Entretiens avec les éducatrices }\end{array}$ \\
\hline Facteurs modérateurs et antécédents & $\begin{array}{l}\text { Entretiens avec les éducatrices } \\
\text { Questionnaires pour les éducatrices }\end{array}$ \\
\hline Réactions des participantes & Entretiens avec les éducatrices \\
\hline Nouvelles connaissances et habiletés & Entretiens avec les éducatrices \\
\hline Transformations dans les pratiques & $\begin{array}{l}\text { Entretiens avec les éducatrices } \\
\text { Segments vidéos d'interactions dans le groupe }\end{array}$ \\
\hline
\end{tabular}

Journal de bord. À partir de ses notes prises dans le journal de bord, l'orthophoniste a rempli des tableaux synthèses pour chaque rencontre avec les éducatrices. Cela a permis de conserver des traces du déploiement du dispositif de développement professionnel auprès de chaque éducatrice : objectifs poursuivis, activités, moments clés des rencontres, sujets abordés et discutés dans les moments de rétroactions. 
Notons que dans cette étude, l'orthophoniste, qui est aussi la première auteure de cet article, jouait également le rôle de chercheuse. En raison de la nature exploratoire du présent projet, cette option a été retenue afin de bénéficier de la relation développée avec les éducatrices, ce qui a permis d'approfondir l'analyse réalisée.

Entretiens semi-dirigés. L'orthophoniste a réalisé trois entretiens semi-dirigés avec les éducatrices : avant le début du projet (1er entretien, $M=28 \mathrm{~min} 45 \mathrm{~s}$ ), pendant (2e entretien, $M=1 \mathrm{~h} 1 \mathrm{~min} 15 \mathrm{~s}$ ) et à la fin de celui-ci (3e entretien, $M=1 \mathrm{~h} 30 \mathrm{~s}$ ). Ces entretiens portaient sur les perceptions des éducatrices à l'égard des retombées de leur participation au dispositif. Deux extraits vidéos, filmés lors de rencontres dans le groupe, ont servi d'amorce pour l'entretien final.

Les entretiens ont été enregistrés, transcrits et segmentés en unité de sens (segments plus ou moins longs se référant à une même idée ; Miles et Huberman, 2003). Chaque unité de sens a été résumée par une paraphrase à l'aide du logiciel d'analyse qualitative MaxQDA. Les paraphrases servent à synthétiser les unités de sens, afin de faciliter leur structuration et leur interprétation, dans le cadre d'une analyse catégorielle selon un modèle mixte (Miles et Huberman, 2003 ; Paillé et Mucchielli, 2012). Deux niveaux d'analyse ont été exécutés parallèlement (Miles et Huberman, 2003) :

1. Un niveau descriptif : les codes du niveau descriptif reflètent la mention d'un de ces éléments dans une unité de sens, soit une pratique de soutien du développement langagier abordée, un volet du dispositif ou un facteur modérateur impliqué, s'il y a lieu. Ainsi, plusieurs codes descriptifs peuvent être attribués à une même unité de sens.

2. Un niveau interprétatif : les codes de ce niveau permettent de lier les témoignages des éducatrices aux trois paliers de retombées intermédiaires du modèle logique de Coldwell et Simkins (2011).

Des tableaux des cooccurrences entre les codes du niveau descriptif et ceux du niveau interprétatif ont facilité les analyses. Trois unités de sens de chaque souscatégorie, soit l'équivalent de $15 \%$ des unités de sens, ont été révisées par la deuxième auteure, spécialiste dans le domaine du développement professionnel (Miles et Huberman, 2003). 
Segments vidéos d'interactions éducatrices/enfants. Des segments vidéos de 7 min $30 \mathrm{~s}$ ont été captés de façon hebdomadaire au cours de la participation au dispositif, durant des moments d'interactions naturels entre les éducatrices et les enfants, selon l'activité en cours : collation, diner, ateliers semi-structurés ou ateliers libres. Douze segments par éducatrice correspondent donc à douze temps de mesure, répartis sur trois périodes : avant (T1 à T3), pendant (T4 à T9) et à la fin (T10 à T12).

Grille d'observation des pratiques de soutien de la communication, du langage et de l'éveil à l'écrit (CLÉÉ). Les segments vidéos ont été analysés à l'aide de la grille CLÉÉ (Bergeron-Morin et al., 2019), une traduction et adaptation du Conversational Responsiveness and Fidelity Tool développé par Friel, Wiggins et Justice (2007). Cet outil permet de recenser l'utilisation de 15 pratiques de soutien du développement langagier à partir de l'analyse de 15 intervalles de 30 secondes. La proportion d'intervalles où chaque pratique est présente est déterminée afin de lui attribuer un score entre 0 et 1 . Un accord interjuges entre la première auteure et une seconde codeuse formée a été réalisé sur $20 \%$ des segments. Les coefficients de Gwet obtenus, qui permettent de vérifier si l'accord interjuges est supérieur à l'accord par chance, sont satisfaisants (entre .63 et .96) (Wongpakaran et al., 2013).

De façon complémentaire à une analyse visuelle des graphiques, des ANOVA à mesures répétées ont été réalisées sur le logiciel $\mathrm{R}$, à l'aide de contrastes qui permettent de vérifier l'effet des trois périodes (avant, pendant, fin) sur l'utilisation de chaque pratique pour les quatre éducatrices. Ces ANOVA à mesures répétées, estimées avec un modèle linéaire mixte, considèrent un effet fixe pour les temps de mesure (T1 à T12) et tiennent compte de la dépendance entre les données d'une même éducatrice à l'aide d'une structure de covariance autorégressive d'ordre 1 . Lorsque le test global du contraste était significatif, des comparaisons multiples ont permis de comparer les périodes entre elles (au seuil de .05, avec correction de Bonferroni). Notons qu'une vérification préalable des postulats de normalité des résidus et d'homogénéité des variances a permis de recourir à un test paramétrique (Milliken et Johnson, 2009). 


\section{Résultats}

Les résultats des analyses sont décrits selon les trois paliers de retombées intermédiaires du modèle de Coldwell et Simkins (2011) : (1) les réactions des participantes ; (2) les nouvelles connaissances et habiletés ; et (3) les transformations dans les pratiques de soutien du développement langagier.

\section{Les réactions des participantes}

Plusieurs commentaires des éducatrices témoignent de leur appréciation quant à leur participation au dispositif de développement professionnel, alors que d'autres pointent vers des impressions plus partagées. Parmi les éléments positifs, elles mentionnent toutes l'alternance entre les moments où elles peuvent observer l'orthophoniste et ceux où elles peuvent mettre à l'essai des pratiques. Cela permet de se «partager le chapeau», comme l'exprime P3 (2e entretien). Elles apprécient également la combinaison de rétroactions sur le vif, au moment où un évènement se produit, et de sessions de rétroaction à postériori, durant lesquelles l'orthophoniste et l'éducatrice reviennent à tour de rôle sur plusieurs moments clés de la rencontre dans le groupe (p. ex., une intervention de l'orthophoniste, une pratique utilisée par l'éducatrice, la réaction d'un enfant).

«J'avais des exemples vraiment concrets. Je trouve que c'est super, parce que ça m'outille davantage. Tu sais, ce n'est pas juste je te donne de l'information pour t'en donner. Je trouve que ça s'applique dans mon présent et ça rend service.» (P1, 2e entretien)

P2 et P4 ajoutent qu'elles ont aimé la souplesse et l'autonomie dans le choix des objectifs à travailler durant la démarche. Les participantes parlent également positivement de la fréquence hebdomadaire des rencontres, puisque cela facilitait l'intégration des pratiques dans la routine et les gardait dans «le feu de l'action» (P4, 3e entretien).

Toutefois, la durée du dispositif est remise en question, en particulier par P3, qui se demande si elle est suffisamment longue pour mener à des transformations dans les pratiques, en particulier auprès d'éducatrices moins expérimentées. Enfin, plusieurs témoignages font référence aux efforts à fournir en début de parcours pour devenir à l'aise avec le regard posé par l'orthophoniste sur leurs pratiques et la caméra. 


\section{De nouvelles connaissances et habiletés}

Le premier constat montre que les éducatrices rapportent peu de nouvelles connaissances directement liées aux pratiques de soutien du développement langagier. Quelques témoignages démontrent tout de même qu'elles ont approfondi leur compréhension de fondements théoriques sous-jacents. Ainsi, P1 explique avoir mieux saisi comment ses commentaires durant le jeu des enfants peuvent nourrir leur imaginaire et leur langage, sans pour autant contrôler leur jeu. Pour sa part, P2 indique avoir mieux compris de quelle façon elle pouvait suivre les initiatives des enfants de façon à influencer leur attention et leur engagement.

Les éducatrices expliquent également certains trucs «pratico-pratiques» $(\mathrm{P} 1,2 \mathrm{e}$ entretien) qu'elles ont retenus : comment décortiquer les longs mots, comment reformuler une phrase à la première personne, comment mettre l'accent sur un son, etc. De plus, toutes les éducatrices nomment une nouvelle prise de conscience des liens qui existent entre différentes pratiques. Par exemple, $\mathrm{P} 2$ réalise qu'en discutant à partir du vécu des enfants, elle s'investit dans des conversations plus longues qui l'amènent à poser davantage de questions. P4 ajoute qu'une écoute sincère des enfants lui permet d'allonger les échanges.

Cependant, la plupart des nouvelles connaissances dont parlent les éducatrices concernent plutôt des facteurs liés au contexte qui facilitent ou entravent l'utilisation des pratiques. Par exemple, P2 dit mieux comprendre comment le type d'activités influence les interactions verbales avec les enfants. Dans une activité dirigée, comme un bricolage, elle constate qu'elle est davantage occupée à structurer l'activité plutôt qu'à être à l'écoute des enfants. Le contexte du jeu libre lui permet davantage d'être à l'écoute et centrée sur le langage des enfants. P1, P3 et P4 déclarent pour leur part avoir découvert le potentiel des autres moments de la routine, comme les collations et les repas, pour soutenir les conversations et «rebondir sur ce qui pop [ressort]» (P3, 3e entretien).

«Ce que j'ai appris le plus, c'est que ça n'a pas besoin d'être avec des outils, ça n'a pas besoin d'être rigide, encadré. Que c'est dans leur vécu, dans ce qu'ils échangent entre eux, quand on peut venir plus supporter, puis rajouter, puis amener de nouveaux éléments !» (P3, 2e entretien) 


\section{Les transformations dans les pratiques}

Les ANOVA à mesures répétées indiquent qu'il ressort des différences significatives entre les périodes pour seulement deux des quinze pratiques analysées, soit «Mettre l'accent sur un mot» $(F=3.79, p=.022)$ et «Utiliser ou amener un enfant à recourir à du langage décontextualisé» $(F=3.68, p=.028)$. Les résultats pour ces deux pratiques sont analysés dans la prochaine section, à la lumière des commentaires des éducatrices. Pour les treize autres pratiques, aucun changement significatif n'est constaté entre les périodes. Dans cette même section, les données quantitatives et qualitatives sont présentées pour trois de ces pratiques, retenues à titre d'exemples, soit «Éviter de dominer la conversation» $(F=2.02, p=.13)$, «Faire un commentaire en réponse à l'enfant» $(F=.86, p=.42)$ et «Reformuler l'énoncé d'un enfant en précisant la prononciation, la syntaxe ou le contenu» $(F=.02, p=.98)$.

Mettre l'accent sur un mot. La Figure 2 montre l'évolution des scores de cette pratique au fil des douze temps de mesure répartis en trois périodes, et ce, pour chaque éducatrice. On constate une augmentation significative entre les périodes avant et pendant pour les quatre éducatrices $(t=2.61, p=.014)$. La différence entre les périodes avant et après se révèle non significative après l'application de la correction de Bonferroni $(t=$ $2.23, p=.033)$.

\section{Figure 2}

Évolution de la pratique «Mettre l'accent sur un mot» au fil de douze temps de mesure répartis en trois périodes

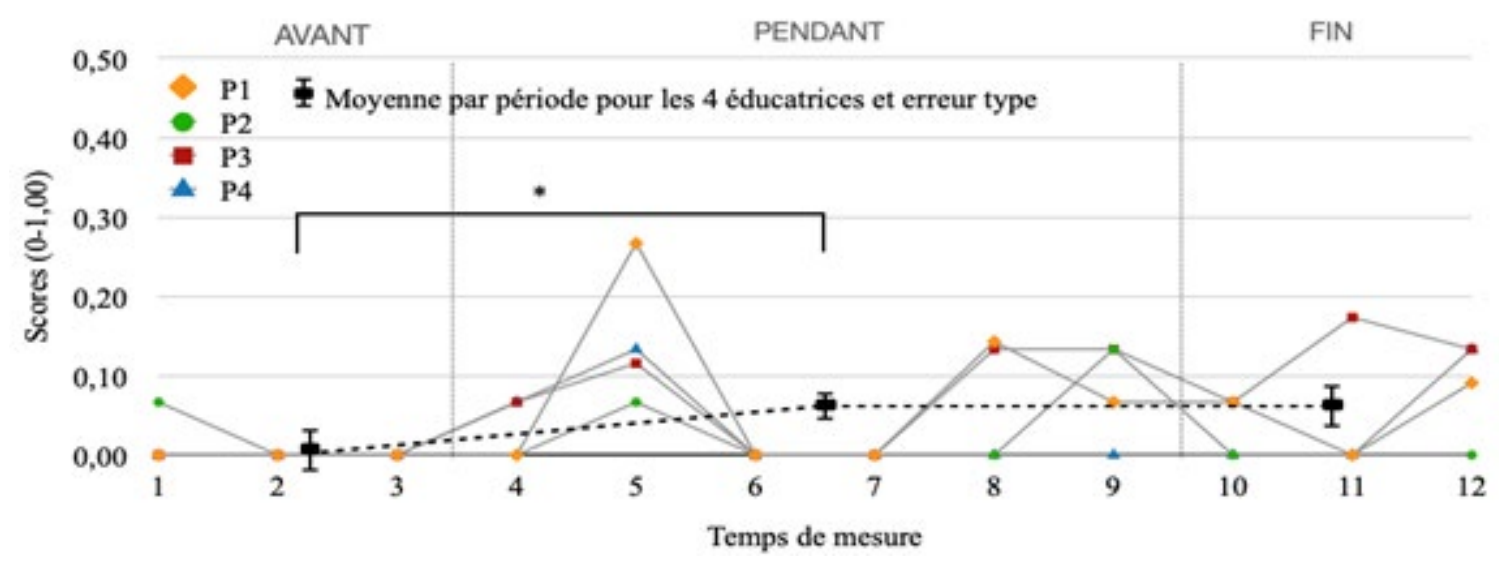

Note. ${ }^{*} p<.05,{ }^{* *} p<.01,{ }^{* * *} p<.001$ 
P3 confirme ce changement dans les entretiens, en soulignant l'importance qu'elle accorde à mettre l'accent sur des mots moins fréquents et à les expliquer aux enfants :

«Dans cette situation, elle dit "chaud" et je lui précise : “C'est un petit peu chaud, on dit : tiède". [...] Depuis notre coanimation, je me dis davantage : "Ah tiens, tu es en train de favoriser la communication, tu es allée enrichir leur conversation, tu es allée leur apprendre un nouveau mot, tu leur as parlé de syllabes".» (P3, 3e entretien)

P1, P2 et P4 mentionnent aussi des changements dans l'utilisation de cette pratique, mais uniquement dans le contexte de la lecture d'histoires.

\section{Utiliser ou amener un enfant à recourir à du langage décontextualisé. La}

Figure 3 montre une différence significative entre les scores des quatre éducatrices lors des périodes avant et fin $(t=2.597, p=.0139)$ pour cette pratique. Toutes les éducatrices mentionnent y accorder de l'attention. C'est «pour amener les enfants à réfléchir», précise P4, ou «pour faire des liens avec leur vécu», dit pour sa part P2. Enfin, P1 rapporte avoir l'impression d'enrichir les conversations en rendant plus explicites des liens complexes entre les idées. P3 précise toutefois que faire des inférences lui semble plus naturel avec certains enfants qu'avec d'autres : «C'est facile avec ces deux-là parce que c'est des enfants qui amènent à ça » (P3, 2e entretien). 


\section{Figure 3}

Évolution de la pratique «Utiliser ou amener un enfant à recourir à du langage décontextualisé» au fil de douze temps de mesure répartis en trois périodes

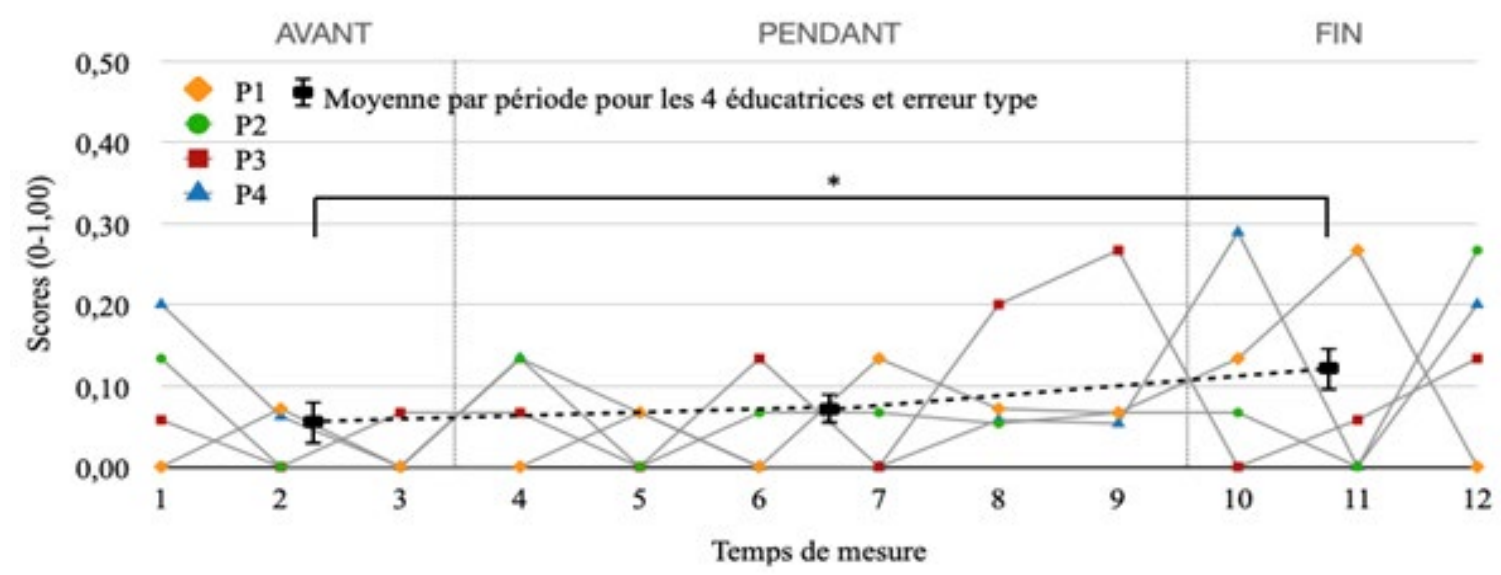

Note. ${ }^{*} p<.05,{ }^{* *} p<.01,{ }^{* * *} p<.001$

Éviter de dominer la conversation. Si la Figure 4 montre une évolution positive de cette pratique, en particulier pour $\mathrm{P} 1$ et $\mathrm{P} 4$, les résultats des ANOVA à mesures répétées n'indiquent pas de différences significatives entre les périodes pour les quatre éducatrices. L'analyse visuelle du graphique montre toutefois qu'un effet de plafonnement pour cette pratique pourrait contribuer à l'absence de changement significatif.

Lors des entretiens, P4 mentionne qu'il s'agissait effectivement d'un défi qu'elle s'était fixé :

«J'ai de la misère avec ça! (rires) Et je pense tout simplement que ça fait partie de ma personnalité. Il n'y a pas beaucoup de silences. C'est quelque chose que je vais travailler. [...] Je dois vraiment à me dire "Ok, attends". [...] Je me rends compte que c'est important pour l'enfant juste le laisser structurer dans sa tête ce qu'il a à me dire, même si ça peut être plus long.» (P4, 2e entrevue)

P3 fait également un constat en ce sens, bien que cette pratique semble déjà maitrisée chez elle : 
«Bien moi, mon plus gros constat, c'est de prendre le temps d'écouter. De me reculer, même quand je suis dans mes souliers d'éducatrice. Mais d'être un peu plus observatrice, de prendre le temps d'attendre. De ne pas toujours nourrir [les échanges] au cas où quelqu'un ne le ferait pas. » (P3, 2e entretien)

\section{Figure 4}

Évolution de la pratique «Éviter de dominer la conversation» au fil de douze temps de mesure répartis en trois périodes

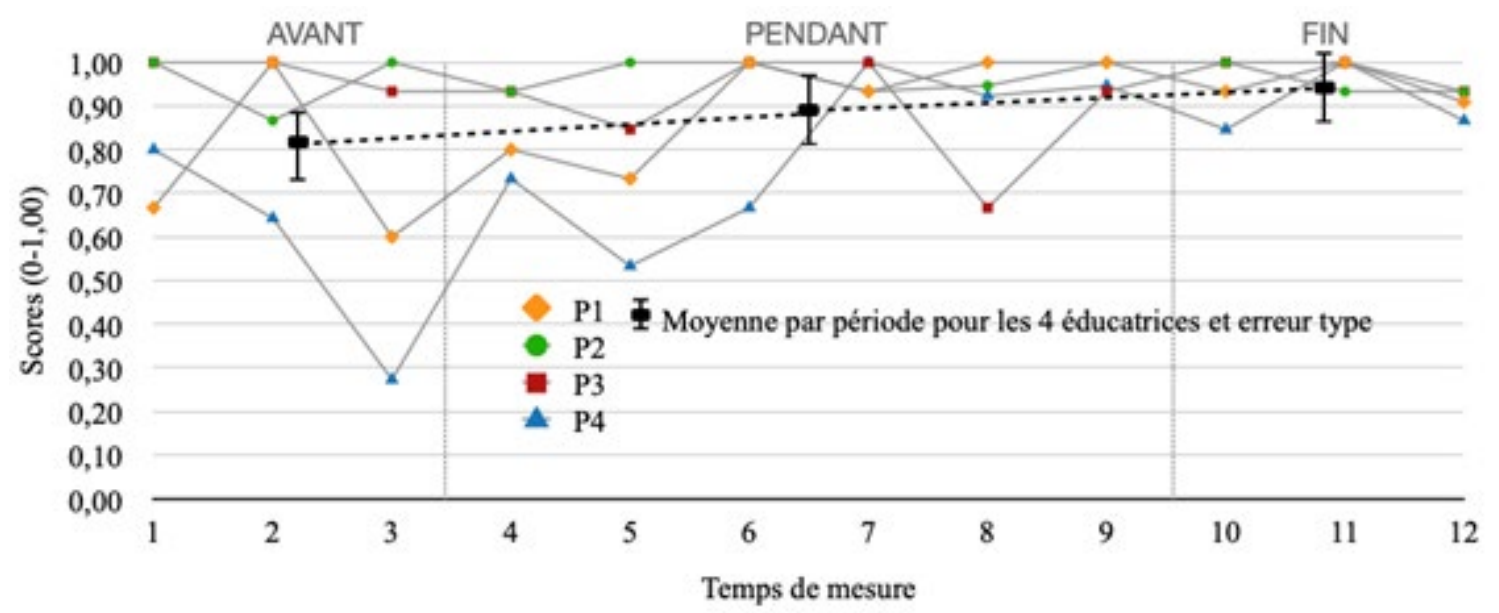

Faire un commentaire en réponse à l'enfant. Comme le montre la Figure 5, cette pratique est utilisée de façon irrégulière entre les éducatrices, d'où l'absence de différences significatives entre les périodes. 


\section{Figure 5}

Évolution de la pratique «Faire un commentaire en réponse à l'enfant» au fil de douze temps de mesure répartis en trois périodes

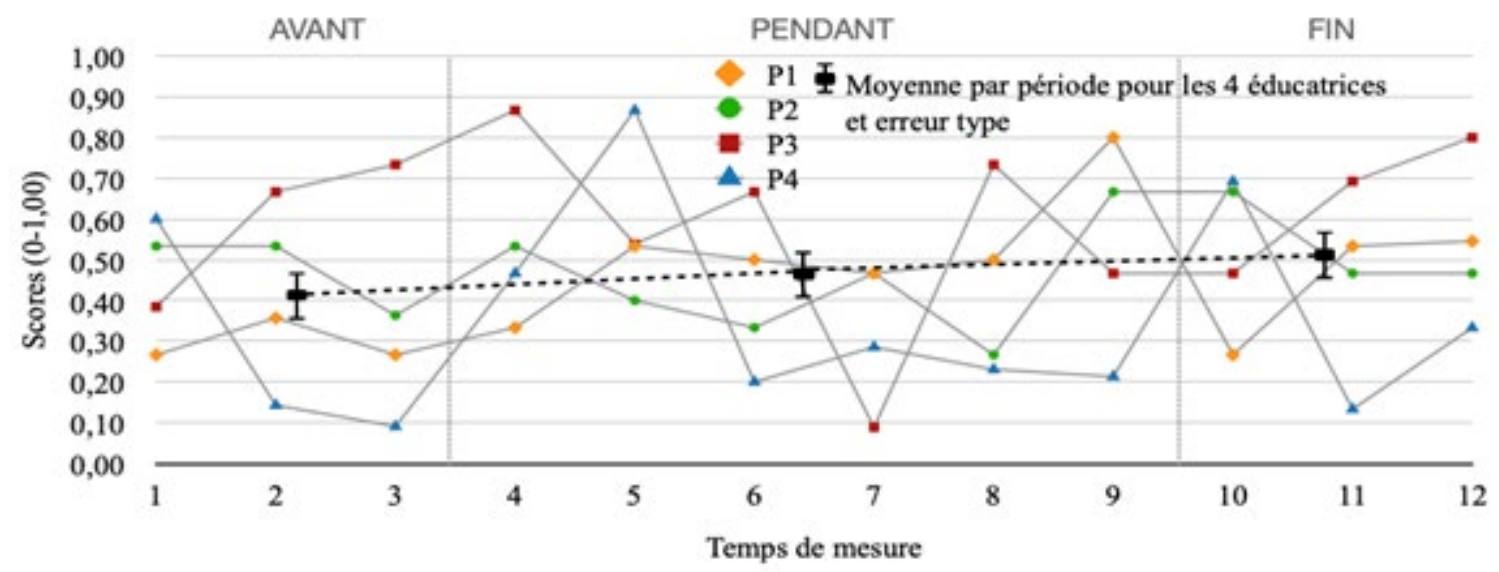

Effectivement, les éducatrices ne mentionnent pas qu'elles commentent davantage les propos de l'enfant, mais indiquent cependant qu'elles le font avec une intentionnalité et une conscience accrues, et dans de nouveaux contextes, comme c'est le cas pour P2 : «Ça ne vient pas aussi naturellement que possible, mais, dès que j’y pense, disons, quand on prend la collation, qu'on s'assoit, j'essaie d'alimenter leurs discussions. Ce n'est pas nécessairement quelque chose que je faisais avant, pendant les collations » (3e entretien).

Il apparait qu'elles utilisent les commentaires principalement dans le but d'allonger les conversations. P4 dit «J'étire la conversation» (P4, 3e entretien), alors que P1 témoigne qu'elle «vient d'ajouter des éléments nouveaux au jeu qui était en cours échafauder - pour faire évoluer le jeu, pour qu'il prenne d'autres dimensions » (P1, 3e entretien).

\section{Reformuler l'énoncé d'un enfant en précisant la prononciation, la syntaxe}

ou le contenu. La reformulation est également une pratique dont l'utilisation est irrégulière entre les temps de mesure, et ce, pour toutes les éducatrices, comme le montre la Figure 6. Seules P1 et P4 mentionnent des transformations dans cette pratique durant les entretiens. Cependant, elles ne parlent pas de changement dans la fréquence des reformulations, mais plutôt dans la spécificité de celles-ci en fonction des énoncés 
produits par des enfants, quant aux sons, aux mots, aux éléments grammaticaux qu'elles accentuent en reformulant la phrase de l'enfant.

«Il y a un enfant qui raccourcit tous les débuts de mots. Ça fait que je prends le temps de vraiment prononcer, de mettre de l'emphase sur le début du mot. Chose que je ne faisais pas du tout avant.» (P1, 2e entretien)

\section{Figure 6}

Évolution de la pratique «Reformuler l'énoncé d'un enfant en précisant la prononciation, la syntaxe ou le contenu» au fil de douze temps de mesure répartis en trois périodes

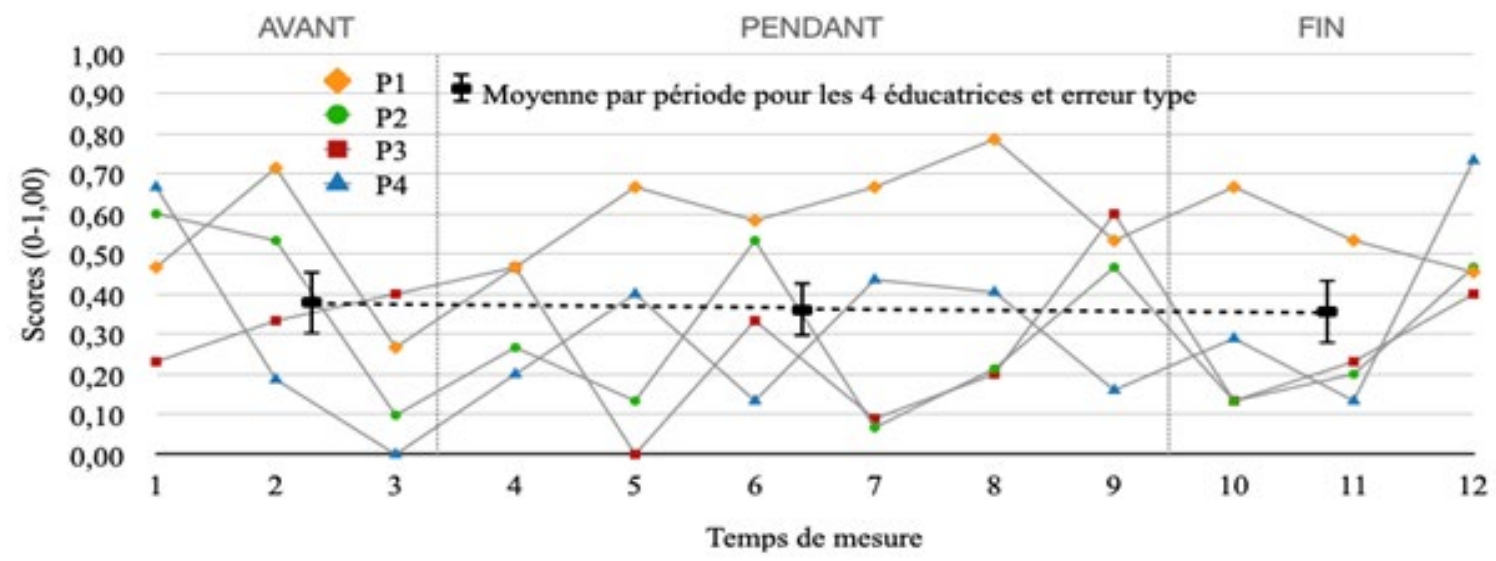

Des variations entre les temps de mesure. L'analyse visuelle des graphiques des quinze pratiques de soutien du développement, à l'instar de ces deux dernières pratiques, montrent la présence de variations en dents de scie entre les temps de mesure, même lors de la période avant qui constitue le niveau de base. À ce sujet, les éducatrices nous indiquent que l'activité en cours, la dynamique du groupe et les tâches à effectuer, qui varient entre les segments vidéos collectés en contexte naturel, influencent la mise en œuvre des pratiques dans leur quotidien. Cependant, à la suite du dispositif de développement professionnel, les éducatrices ajoutent qu'elles sont en mesure d'utiliser les pratiques de soutien du développement langagier dans une plus grande variété d'activités et de façon plus intégrée à leur routine quotidienne. P4 dit par exemple être plus à l'affut pour saisir les occasions propices, celles qui se présentent naturellement, pour engager des conversations avec les enfants : «Comment dire... Je suis plus vive 
d'esprit pour me dire : “Oh ! ok ! il m'ouvre une porte, ça fait que prends la porte, essaie de communiquer à ce moment-là."” (P4, 3e entretien). Pour ce faire, il s'avère nécessaire pour les éducatrices de prioriser ces moments vis-à-vis des autres tâches inhérentes au rôle d'éducatrice en CPE (p. ex., préparer la collation, organiser les lits pour la sieste, etc.).

\section{Discussion}

L'utilisation du modèle logique de Coldwell et Simkins (2011) a permis de décortiquer les retombées de la participation au dispositif de développement professionnel selon trois paliers intermédiaires : (1) les réactions des éducatrices ; (2) les nouvelles connaissances et habiletés développées; et (3) les transformations dans leurs pratiques de soutien du développement langagier, et de les mettre en relation avec certains facteurs modérateurs et antécédents.

Les résultats montrent d'abord que, malgré les critères d'efficacité considérés dans le présent dispositif de développement professionnel, les transformations dans leurs connaissances et leurs pratiques demeurent modestes, confirmant les résultats obtenus par plusieurs autres études (p. ex., Markussen-Brown et al., 2017 ; McDonald et al., 2015 ; Piasta et al., 2012; Wasik et Hindman, 2014). Cependant, grâce au regard transversal que pose la présente étude, grâce au devis mixte et au modèle logique, différentes pistes de réflexion peuvent être abordées face à ce constat.

\section{L'éclairage apporté par les réactions des éducatrices}

Le développement de la relation entre l'orthophoniste et les éducatrices transparait dans le discours de ces dernières. Les rôles de chacune se sont négociés et les attentes mutuelles se sont précisées au fil des rencontres. La difficulté des éducatrices de parler de leurs propres pratiques complexifie la possibilité de vérifier à quel point la dynamique relationnelle qui s'est établie a permis aux éducatrices d'y faire valoir leur propre expertise et d'y développer leur pratique réflexive. Il serait donc particulièrement intéressant d'étudier davantage les stratégies utilisées par l'orthophoniste pour jouer ce rôle d'accompagnement, auquel la formation initiale en orthophonie ne la prépare pas nécessairement (Law et al., 2012 ; Neuman et Wright, 2010). 
Malgré tout, on constate que les moments clés rapportés lors des entretiens par les éducatrices montrent qu'elles ont toutes bâti, au fil des rencontres, un vécu et des références communes avec l'orthophoniste. Cela a permis d'ancrer le contenu proposé dans des exemples concrets et spécifiques au contexte de travail de l'éducatrice, augmentant ainsi la cohérence du dispositif (Schachter et al., 2016), même si ces épisodes peuvent s'avérer difficiles à planifier et à reproduire.

Cependant, les propos des éducatrices nous informent peu concernant l'influence de leurs antécédents sur leurs réactions. En effet, différentes caractéristiques du dispositif proposé, par exemple l'accompagnement réflexif ou la durée, pourraient avoir des retombées différentes selon l'expérience de travail et la formation initiale des éducatrices. De même, les données recueillies nous offrent peu de perspectives au sujet de l'influence d'autres facteurs au sein de l'organisation (p. ex., changements dans les tâches des éducatrices, orientations du CPE) sur leurs réactions face au dispositif de développement professionnel.

\section{Vers de nouvelles connaissances et habiletés}

Les résultats soulignent que les éducatrices rapportent peu de nouvelles connaissances portant sur les pratiques elles-mêmes, alors que le contenu ciblé les concernait directement. Or, les éducatrices parlent plus abondamment de leur meilleure compréhension des facteurs contextuels qui influencent l'utilisation de ces pratiques dans leur quotidien. Elles disent, par exemple, mieux comprendre quels contextes leur permettent plus facilement de mettre de côté leurs autres tâches pour saisir les occasions de communication qu'offrent les enfants.

Développer des connaissances et des habiletés sur la façon d'intégrer le soutien du développement langagier aux autres rôles d'une éducatrice (p. ex., préparer les lits, servir les repas, changer les couches, etc.) peut ainsi s'avérer aussi essentiel que développer celles sur les pratiques en soi (Burchinal, 2018). Cela peut cependant nécessiter l'ajout de modalités de développement professionnel qui permettent les échanges et la concertation entre collègues et avec la direction, afin qu'elles y trouvent des pistes de solutions (Zaslow et al., 2010). À cet égard, Urban et al. (2011) avancent que les compétences du personnel éducateur en petite enfance ne peuvent s'actualiser que dans un système 
lui-même compétent. Il serait donc essentiel que l'équipe d'un même CPE soit impliquée dans l'ensemble du dispositif de développement professionnel (Zaslow et al., 2010).

Effectivement, comme l'avancent Markussen-Brown et al. (2017) et Schachter et al. (2016), différents types de connaissances doivent être considérés dans la mise en œuvre et l'évaluation d'un dispositif de développement professionnel : des connaissances plus théoriques sur les pratiques de soutien du développement, mais également des connaissances pratiques ancrées dans la réalité des éducatrices, à savoir comment les mettre en œuvre dans leur contexte précis.

\section{Des façons de mobiliser les connaissances et habiletés dans la pratique quotidienne des éducatrices}

Les résultats amènent aussi à se questionner sur la façon de transformer des pratiques dans cette réalité professionnelle complexe et exigeante. Cette question est au cœur de la présente étude, mais également de tout le champ de recherche sur le développement professionnel en éducation (Schachter et al., 2019). Si la présente étude fait ressortir peu de transformations quantitatives dans les pratiques à la suite de la participation des éducatrices au dispositif de développement professionnel (Markussen-Brown et al., 2017 ; Wasik et Hindman, 2014), elle ouvre néanmoins sur des transformations plus qualitatives que ces dernières mentionnent avoir amorcées, mais qui ne se traduisent pas encore dans les interactions observées dans le groupe. L'analyse selon les différents paliers du modèle logique de Coldwell et Simkins (2011) permet d'envisager certaines hypothèses à cet égard.

D'une part, les résultats obtenus réitèrent l'importance d'ancrer davantage les nouvelles connaissances et habiletés des éducatrices quant aux pratiques de soutien du développement langagier dans leur mise en œuvre au quotidien en CPE. En effet, les variations constatées entre les temps de mesure laissent présager l'influence de facteurs modérateurs, comme les autres tâches de l'éducatrice, l'activité en cours ou les enfants présents dans l'interaction, dans les effets du dispositif sur les pratiques (Chen et de Groot, 2014 ; Parent et Bouchard, 2020). L'intérêt du modèle logique de Coldwell et Simkins (2011), par rapport à d'autres modèles similaires (voir Boylan et al., 2018), réside précisément dans la place qu'il accorde à l'influence de ces facteurs modérateurs. Il 
serait donc hautement pertinent d'approfondir notre connaissance de ceux-ci, notamment en identifiant différentes catégories de facteurs modérateurs dans le modèle.

D'autre part, la présente étude témoigne de l'intérêt de recourir à des données qualitatives pour décrire les changements dans l'utilisation des pratiques, pour faire ressortir ou préciser d'autres éléments que des outils de mesure quantitatifs ne permettent pas de dégager. En effet, dans les résultats de l'étude, il apparait, à travers les propos des éducatrices, que bonifier la qualité du soutien au développement langagier ne signifie pas toujours faire plus, mais aussi faire mieux. Cela peut s'exprimer dans la sélection d'une pratique à utiliser à un moment donné et dans l'ajustement de celle-ci face à un enfant en particulier. P1 nous en donne un exemple lorsqu'elle parle de la précision accrue des reformulations pour certains enfants. P3 souligne pour sa part sa vigilance accrue pour amener certains enfants à recourir au langage décontextualisé. Faire mieux pour le soutien du langage des enfants peut aussi signifier en dire moins, en laissant davantage de place aux enfants dans les conversations. Wasik et Hindman (2018) soulignent d'ailleurs le potentiel des pauses et des silences, particulièrement pour les enfants plus fragiles sur le plan langagier. Par conséquent, pour ce faire, il peut être nécessaire de réduire l'utilisation de certaines pratiques, comme les questions et les commentaires.

Finalement, l'éducatrice doit également être en mesure de choisir les moments les plus opportuns pour construire des échanges avec les enfants, lorsqu'ils sont engagés et intéressés, soit, comme le dit P4, «prendre la porte que l'enfant nous ouvre» pour amorcer une conversation de plusieurs tours. Comme mentionné précédemment, pour ce faire, il est nécessaire que chaque éducatrice parvienne à saisir les facteurs modérateurs qui influencent sa capacité à saisir ces occasions.

\section{Les limites et les perspectives de recherche}

Certaines limites sont à considérer dans l'interprétation de ces résultats. D'abord, un biais potentiellement causé par l'accent mis, dans le dispositif, sur la modalité de cointervention, qui a pu être au détriment de l'attention portée aux autres modalités, est à considérer. Aussi, le double chapeau de l'orthophoniste/étudiante-chercheuse, malgré une vigilance à cet égard, a pu amener un biais dans les réponses des éducatrices, en lien avec la désirabilité sociale, et dans l'interprétation de celles-ci. Notons également que le développement et la validation de l'outil de mesure des pratiques de soutien du 
développement langagier devraient être poursuivis afin de prendre davantage en compte la qualité de ces pratiques, en plus de leur quantité.

Aussi, bien sûr, le petit nombre de participants a limité les méthodes d'analyse statistique qui ont pu être utilisées et rend impossible une généralisation des résultats. De plus, des caractéristiques des éducatrices (p. ex., leur formation et expérience), si elles permettent de brosser des portraits contrastés, n'ont pas été suffisamment détaillées pour être mises en lien avec les autres données recueillies, ce qui en limite la portée.

Enfin, force est de constater que le modèle de Coldwell et Simkins (2011) simplifie une réalité complexe en laissant des zones d'ombre entre les différents paliers de retombées intermédiaires. De plus, si son utilisation a permis de structurer les analyses, chaque composante du modèle pourrait être décrite de façon plus systématique dans le devis de recherche, en ciblant, par exemple, différents types de connaissances et d'habiletés développés. De même, les facteurs modérateurs et antécédents des participantes gagneraient à faire l'objet d'une collecte de données plus exhaustive afin de mieux décrire leur influence sur les retombées intermédiaires.

\section{Conclusion}

Cette étude a permis de décrire les retombées intermédiaires d'un dispositif de développement professionnel portant sur le soutien du développement langagier, à partir du modèle logique de Coldwell et Simkins (2011). Si peu de changements sur la fréquence de l'utilisation des pratiques observées sont ressortis, les propos des éducatrices montrent néanmoins qu'elles ont acquis de nouvelles connaissances sur les contextes favorables à l'utilisation de ces pratiques, et amorcé certaines transformations. L'étude met en relief l'importance de considérer l'influence des facteurs modérateurs, propres au contexte de mise en œuvre dans les groupes en CPE, tout au long d'un tel dispositif de développement professionnel. Elle ouvre aussi la porte à de nouvelles avenues de recherche pour explorer davantage le rôle de l'orthophoniste, comme accompagnatrice dans le dispositif, de même que les retombées sur son propre parcours professionnel. Des pistes sont également avancées pour continuer de raffiner le dispositif de développement professionnel proposé, de même que l'utilisation du modèle logique retenu. 


\section{Références}

Barnes, E. M. et Dickinson, D. K. (2017). The relationship of Head Start teachers' academic language use and children's receptive vocabulary. Early Education and Development, 28(7), 794-809. https://doi.org/10.1080/10409289.2017.1340069

Bergeron-Morin, L. (2017). Rapport d'évaluation de l'action «Je te comprends, je te parle» 2014-2017. Table de concertation Filons Jeunesse-Avenir d'enfants.

Bergeron-Morin, L., Bouchard, C., Julien, C., Parent, A.-S., Sylvestre, A. et Di Sante, M. (2019). Grille d'observation des pratiques éducatives en soutien à la Communication, au Langage et à l'Éveil à l'Écrit (CLÉÉ) [document inédit]. Faculté des sciences de l'éducation, Université Laval.

Bouchard, C., Bigras, N., Cantin, G., Coutu, S., Blain-Brière, B., Eryasa, J., Charron, A. et Brunson, L. (2010). Early childhood educators' use of language-support practices with 4-year-old children in child care centres. Early Childhood Education Journal, 37(5), 371-379. https://doi.org/10.1007/s10643-009-0355-7

Boylan, M., Coldwell, M., Maxwell, B. et Jordan, J. (2018). Rethinking models of professional learning as tools: a conceptual analysis to inform research and practice. Professional Development in Education, 44(1), 120-139. https://doi.org/ $\underline{10.1080 / 19415257.2017 .1306789}$

Burchinal, M. (2018). Measuring early care and education quality. Child Development Perspectives, 12(1), 3-9. https://doi.org/10.1111/cdep.12260

Chen, J. J. et de Groot, K. S. (2014). The quality of teachers' interactive conversations with preschool children from low-income families during small-group and largegroup activities. Early Years, 34(3), 271-288. https://doi.org/10.1080/09575146.2 $\underline{014.912203}$

Coldwell, M. (2019). Reconsidering context: six underlying features of context to improve learning from evaluation. Evaluation, 25(1), 99-117. https://doi. org/10.1177/1356389018803234

Coldwell, M. et Simkins, T. (2011). Level models of continuing professional development evaluation: a grounded review and critique. Professional Development in Education, 37(1), 143-157. https://doi.org/10.1080/19415257.2010.495497 
Corbière, M. et Larivière, N. (2014). Méthodes qualitatives, quantitatives et mixtes : dans la recherche en sciences humaines, sociales et de la santé. Presses de l'Université du Québec.

Dickinson, D. K. (2011). Teachers' language practices and academic outcomes of preschool children. Science, 333(6045), 964-967. https://doi.org/10.1126/ $\underline{\text { science. } 1204526}$

Dockrell, J. E., Bakopoulou, I., Law, J., Spencer, S. et Lindsay, G. (2015). Capturing communication supporting classrooms: The development of a tool and feasibility study. Child Language Teaching and Therapy, 31(3), 271-286. https://doi. org/10.1177/0265659015572165

Duncan, G. J., Claessens, A., Huston, A. C., Pagani, L. S., Engel, M., Sexton, H., Dowsett, C. J., Magnuson, K., Klebanov, P., Feinstein, L., BrooksGunn, J., Duckworth, K. et Japel, C. (2007). School readiness and later achievement. Developmental Psychology, 43(6), 1428-1446. https://doi. org/10.1037/0012-1649.43.6.1428

Dunst, C. J. et Trivette, C. M. (2009). Let's be PALS: An evidence-based approach to professional development. Infants \& Young Children, 22(3), 164-176. https://doi. org/https://doi.org/10.1097/IYC.0b013e3181abe169

Friel, S., Wiggins, A. et Justice, L. (2007). Conversational Responsiveness Assessment and Fidelity Tool (CRAFT) [document inédit]. University of Virginia.

Fukkink, R. G. et Lont, A. (2007). Does training matter? A meta-analysis and review of caregiver training studies. Early Childhood Research Quarterly, 22(3), 294-311. https://doi.org/10.1016/j.ecresq.2007.04.005

Girolametto, L. et Weitzman, E. (2002). Responsiveness of child care providers in interactions with toddlers and preschoolers. Language, Speech, and Hearing Services in Schools, 33(4), 268-281. https://doi.org/10.1044/0161-1461(2002/022)

Girolametto, L., Weitzman, E. et Greenberg, J. (2006). Facilitating language skills: Inservice education for early childhood educators and preschool teachers. Infants \& Young Children, 19(1), 36-49. https://doi. org/10.1097/00001163-200601000-00005 
Hoff, E. (2003). The specificity of environmental influence: socioeconomic status affects early vocabulary development via maternal speech. Child Development, 74(5), 1368-1378. https://doi.org/10.1111/1467-8624.00612

Justice, L. M., Jiang, H. et Strasser, K. (2018). Linguistic environment of preschool classrooms: What dimensions support children's language growth? Early Childhood Research Quarterly, 42(1), 79-92. https://doi.org/10.1016/j. ecresq.2017.09.003

Law, J., Beecham, J. et Lindsay, G. (2012). Effectiveness, costing and cost effectiveness of interventions for children and young people with speech, language and communication needs (DFE-RR247-BCRP5) [Rapport de recherche]. https:// www.bettercommunication.org.uk/BCRP/DFE-RR247-BCRP5.pdf

Leithwood, K. et Levin, B. (2005). Assessing school leader and leadership. Programme effects on pupil learning (RR662) [Rapport de recherche]. Department for Education and Skills, Ontario, Canada.

LoCasale-Crouch, J., Konold, T. R., Pianta, R. C., Howes, C., Burchinal, M., Bryant, D., Clifford, R., Early, D. et Barbarin, O. (2007). Observed classroom quality profiles in state-funded pre-kindergarten programs and associations with teacher, program, and classroom characteristics. Early Childhood Research Quarterly, 22(1), 3-17. https://doi.org/10.1016/j.ecresq.2006.05.001

Markussen-Brown, J., Juhl, C. B., Piasta, S. B., Bleses, D., Højen, A. et Justice, L. M. (2017). The effects of language- and literacy-focused professional development on early educators and children: A best-evidence meta-analysis. Early Childhood Research Quarterly, 38(1), 97-115. https://doi.org/10.1016/j.ecresq.2016.07.002

McDonald, D., Proctor, P., Gill, W., Heaven, S., Marr, J. et Young, J. (2015). Increasing early childhood educators' use of communication-facilitating and language-modelling strategies: brief speech and language therapy training. Child Language Teaching and Therapy, 31(3), 305-322. https://doi. org/10.1177/0265659015588203

Miles, M. B. et Huberman, H. S. (2003). Analyse des données qualitatives. De Boeck Université. 
Milliken, G. A. et Johnson, D. E. (2009). Analysis of repeated measures experiments when the ideal conditions are not satisfied. Dans G. A. Milliken et D. E. Johnson (dir.), Analysis of messy data (2e éd., p. 535-571). CRC Press.

Neuman, S. B. et Wright, T. S. (2010). Promoting language and literacy development for early childhood educators: A mixed-methods study of coursework and coaching. The Elementary School Journal, 111(1), 63-86. https://doi.org/10.1086/653470

Paillé, P. et Mucchielli, A. (2012). L'analyse qualitative en sciences humaines et sociales (3e éd.). Armand Colin.

Pampalon, R. P., Hamel, D. et Philibert, M. (2004). Inégalités sociales et services de proximité au Québec - Développement d'un système d'évaluation issu d'une collaboration entre chercheurs et intervenants. Santé, Société et Solidarité, (2), 73-88. https://doi.org/10.3406/oss.2004.996

Parent, A.-S. et Bouchard, C. (2020). Pratiques enseignantes pour soutenir le langage oral des enfants selon les contextes de classe à l'éducation préscolaire 5 ans. Initio, 8(1), 37-55. https://hal.archives-ouvertes.fr/INITIO/hal-02955343

Peleman, B., Lazzari, A., Budginaite, I., Siarova, H., Hauari, H., Peeters, J. et Cameron, C. (2018). Continuous professional development and ECEC quality: Findings from a European systematic literature review. European Journal of Education, 53(1), 9-22. https://doi.org/10.1111/ejed.12257

Piasta, S. B., Justice, L. M., Cabell, S. Q., Wiggins, A. K., Turnbull, K. P. et Curenton, S. M. (2012). Impact of professional development on preschool teachers' conversational responsivity and children's linguistic productivity and complexity. Early Childhood Research Quarterly, 27(3), 387-400. https://doi.org/10.1016/j. ecresq.2012.01.001

Scarinci, N., Rose, T., Pee, J. et Webb, K. (2015). Impacts of an in-service education program on promoting language development in young children: A pilot study with early childhood educators. Child Language Teaching and Therapy, 31(1), 37-51. https://doi.org/10.1177/0265659014537508

Schachter, R. E. (2015). An analytic study of the professional development research in early childhood education. Early Education and Development, 26(8), 1057-1085. https://doi.org/10.1080/10409289.2015.1009335 
Schachter, R. E., Gerde, H. K. et Hatton-Bowers, H. (2019). Guidelines for selecting professional development for early childhood teachers. Early Childhood Education Journal, 47(4), 395-408. https://doi.org/10.1007/s10643-019-00942-8

Schachter, R. E., Spear, C. F., Piasta, S. B., Justice, L. M. et Logan, J. A. R. (2016). Early childhood educators' knowledge, beliefs, education, experiences, and children's language- and literacy-learning opportunities: What is the connection? Early Childhood Research Quarterly, 36(3), 281-294. https://doi.org/10.1016/j. ecresq.2016.01.008

Sylvestre, A., Bouchard, C., Beaulieu, K., Déry, É., Grégoire., L., Savard, I. et Leboeuf, M. (2016, avril). COmmunauté - Parents - Enfants (COPE). Unis pour le développement langagier des enfants [Rapport de recherche]. Fonds de Soutien à l'innovation Sociale (FSIS), Université Laval. https://archipel.uqam.ca/12025/1/ sylvestre\%20et\%20al_2016_COPE.pdf

Sylvestre, A. et Mérette, C. (2012). Language delay in severely neglected children: A cumulative or specific effect of risk factors? Child Abuse \& Neglect, 34(6), 414428. https://doi.org/10.1016/j.chiabu.2009.10.003

Urban, M., Vandenbroeck, M., Lazzari, A., Peeters, J. et Van Laere, K. (2011). CoRe: Competence requirements in early childhood education and care: Final report [Rapport de recherche]. Direction générale Éducation et culture, Commission européenne. https://repository.uel.ac.uk/item/8618y

Wasik, B. A. et Hindman, A. H. (2011). Identifying critical components of an effective preschool language and literacy coaching intervention. Dans S. B. Neuman et D. K. Dickinson (dir.), Handbook of early literacy research, volume 3 (p. 322-336). Guilford Press.

Wasik, B. A. et Hindman, A. H. (2013). Realizing the promise of open-ended questions. The Reading Teacher, 67(4), 302-311. https://doi.org/10.1002/trtr.1218

Wasik, B. A. et Hindman, A. H. (2014). Understanding the active ingredients in an effective preschool vocabulary intervention: An exploratory study of teacher and child talk during book reading. Early Education and Development, 25(7), 10351056. https://doi.org/10.1080/10409289.2014.896064 
Wasik, B. A. et Hindman, A. H. (2018). Why wait? The importance of wait time in developing young students' language and vocabulary skills. The Reading Teacher, 72(3), 369-378. https://doi.org/10.1002/trtr.1730

Wayne, A. J., Yoon, K. S., Zhu, P., Cronen, S. et Garet, M. S. (2008). Experimenting with teacher professional development: Motives and methods. Educational Researcher, 37(8), 469-479. https://doi.org/10.3102/0013189x08327154

Wongpakaran, N., Wongpakaran, T., Wedding, D. et Gwet, K. L. (2013). A comparison of Cohen's Kappa and Gwet's AC1 when calculating inter-rater reliability coefficients: A study conducted with personality disorder samples. BMC Medical Research Methodology, 13, Article 61, 1-7. https://doi. org/10.1186/1471-2288-13-61

Zaslow, M., Tout, K., Halle, T., Whittaker, J. V. et Lavelle, B. (2010). Toward the identification of features of effective professional developmental for early childhood educators: Literature review. U.S. Department of Education. https:// www2.ed.gov/rschstat/eval/professional-development/literature-review.pdf 